\title{
Diseño de un instrumento de bajo costo para la calibración de micromanómetros diferenciales
}

\author{
Design of a low cost device for the calibration \\ of differential micromanometer
}

\begin{abstract}
Constantino, Pablo (1), Camacho, Javier (2), Maciel, Andrés (1), Acquarone, Alejandro (1).
(1)Departamento de Metrología, Laboratorio Tecnológico del Uruguay, LATU - (2) VIA University College Denmark.
\end{abstract}

Contacto: pconstan@latu.org.uy

RECIBIDO: 21/5/2014 - APROBADO: 26/11/2014

\begin{abstract}
Resumen
Este artículo describe el diseño y construcción de un prototipo de sistema primario basado en el sistema de campana invertida para la calibración de medidores de presión diferencial de rangos bajos. Se discute la construcción del instrumento, se realiza una caracterización dimensional y se efectúa la calibración de un sensor diferencial electrónico, comparando estos resultados con los obtenidos por el Centro Español de Metrología (CEM) para el mismo instrumento.

Palabras clave: Metrología, presión, campana invertida, presión diferencial, micromanómetro.
\end{abstract}

\begin{abstract}
This paper describes the design and development of a primary system prototype based on the diving bell manometer, for the calibration of low range differential pressure gauges. Construction of the instrument is discussed, and a basic dimensional characterization is accomplished. With this prototype a digital differential sensor calibration is performed, and then compared these results with those obtained by the Spanish National Metrology Institute (CEM) for the same instrument.

Keywords: Metrology, pressure, diving bell, differential manometer, micromanometer.
\end{abstract}

\section{Introducción}

Cada vez es más frecuente en Uruguay la necesidad de calibrar equipos medidores de intervalos ultra bajos de presión, del orden de 1 a $3 \mathrm{kPa}$ en plena escala. Si bien son instrumentos que existen en el mercado hace años, las aplicaciones existentes eran mayormente para reducción de costos en sistemas de aire acondicionado, control de fugas en la industria o similares, para los cuales las exigencias en exactitud $u$ otras condiciones metrológicas de los instrumentos no eran muy demandantes. En estos últimos años, en la medida que más laboratorios uruguayos implementan sistemas de calidad consistentes con ISO/IEC 17025 (International Organization for Standarization, 2005), comienza a surgir la necesidad de contar con patrones adecuados para satisfacer la demanda de calibración de estos instrumentos. Las aplicaciones de micromanómetros diferenciales son variadas, al igual que las precisiones y tipos de instrumentos disponibles. Además de las aplicaciones mencionadas, se utilizan en equipos médicos, salas de presurización o certificación de salas limpias, donde deben existir atmósferas controladas que permitan establecer condiciones adecuadas de trabajo y baja contaminación.

La dificultad principal en la calibración de este tipo de instrumentos de intervalos tan bajos de presión es que la incertidumbre relativa aportada por los patrones normales suele ser excesiva en relación a la resolución de los mismos.
Cuando se requieren calibraciones de alta precisión, se deben utilizar patrones de tipo fundamental, que pueden ser balanzas diferenciales de presión que constan de dos ensambles pistón-cilindro dispuestos en la misma plataforma. También es posible conectar dos balanzas con ensambles pistón-cilindro similares a las entradas de baja y alta presión del instrumento diferencial. En ambos casos se realizan pequeñas variaciones de masa en uno de los ensambles para efectuar la comparación a lo largo del intervalo de lectura del instrumento.

Debido al alto costo de los patrones fundamentales de pistón-cilindro, este sistema de medida resulta inadecuado para la actual demanda existente en Uruguay, por lo que se propone buscar una solución alternativa que permita obtener incertidumbres de calibración adecuadas a las necesidades de los laboratorios locales, sin realizar inversiones tan elevadas. Por otra parte, la utilización de patrones digitales tampoco representa una solución a la demanda, ya que los bajos intervalos de confirmación metrológica para equipos digitales y la necesidad de calibrar los mismos en laboratorios del exterior implica necesariamente la intermitencia del servicio a lo largo del año.

El objetivo de este trabajo es el desarrollo y la construcción de un prototipo de bajo costo basado en el sistema de Campana invertida (o campana sumergida), que pueda ser caracterizado dimensionalmente en el laboratorio de Longitud del Laboratorio Tecnológico del Uruguay (LATU). Con 
este prototipo se realizan mediciones de un equipo digital para verificar el correcto funcionamiento de este sistema modificado y se evalúan mejoras y correcciones al diseño que permitan desarrollar un instrumento final capaz de medir presiones diferenciales con exactitud e incertidumbre adecuadas.

\section{Materiales y Métodos}

\section{Sistema de campana invertida}

Este antiguo sistema mecánico de medición de presión diferencial, hoy en desuso, consta de dos campanas de acero inoxidable invertidas, sumergidas parcialmente en un líquido y suspendidas de la palanca o brazo de una balanza de dos platos, funcionando en equilibrio. Al variar la cantidad de gas dentro de una de las campanas, este desplaza el líquido interior generando una fuerza de empuje positiva o negativa que debe equilibrarse con pesos en la balanza. El área efectiva de la campana, obtenida mediante la calibración dimensional y la diferencia de masa entre los platos, determina la diferencia de presión entre una y otra campana según la ecuación:

$\Delta P=\frac{m \cdot g_{l}}{A}$

Con: $m$ la masa agregada, $g_{l}$ la gravedad local y $A$ el área efectiva de la campana.

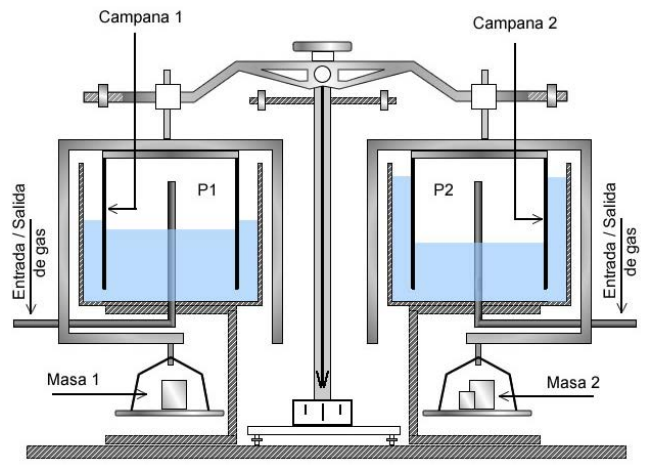

Figura 1. Diagrama de balanza de dos campanas.

Si bien este es el sistema básico, se han propuesto también sistemas en los que las campanas están conectadas a una balanza de torsión y la lectura en unidades de presión se realiza en forma directa (Rostron Hindley, 1947).

En algunos laboratorios nacionales existen aún hoy en servicio balanzas de campana simple, donde una sola campana es suspendida de uno de los brazos de una balanza de dos platos, mientras que el otro se conserva con un plato simple, equilibrado con masas. La variación de masas en uno y otro plato determina el diferencial de presión positivo y negativo.

Aunque este tipo de equipos se ha dejado de fabricar, la evolución de la metrología en las últimas décadas permite realizar mediciones dimensionales y de masa con mucha facilidad y precisión, por lo que algunas características pueden ser simplificadas y modernizadas para mejorar el instrumento tradicional.

El diseño propuesto es un sistema de campana de tipo simple (una sola campana) suspendido de una balanza digital en la cual se leerán las diferencias de masa.

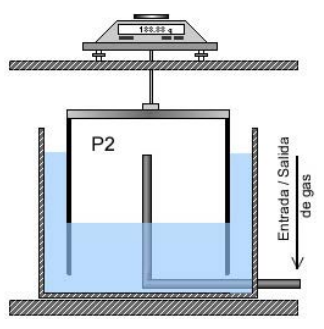

Figura 2. Diagrama del prototipo propuesto.

Elemento sensor. Como elemento sensor (cilindro de campana) se requiere utilizar un cilindro recto, en lo posible sin costuras ni deformidades visibles en el extremo cerrado que puedan incrementar la incertidumbre en la medida del área específica en forma dimensional. El largo de este elemento también es importante, ya que esta magnitud determinará el intervalo de medición de la balanza. Luego de varias propuestas se decide utilizar una sección de termo de acero inoxidable, cortado con un cortador de tubos.

Depósito. El depósito contenedor de fluido dentro del cual estará sumergida parcialmente la campana invertida debe tener convenientemente un diámetro cercano al de la campana. Por otra parte, se prioriza la transparencia del mismo para permitir la visualización del comportamiento del instrumento en servicio. Para esto se utiliza un cilindro de vidrio con una perforación a través de la cual se introducirá un tubo de acero inoxidable con el que se variará la presión del sistema. Para simplificar el modelo, este mismo tubo oficiará de venteo para el equilibrio inicial del instrumento. Como fluido se utiliza agua destilada.

Instrumentos de medición. La caracterización del instrumento depende básicamente de la medición del diámetro interior de la campana a partir del cual se calculará el área específica. Debido a la geometría del instrumento, no se cuenta en el LATU con patrones adecuados para medir el diámetro interior en forma directa, por tanto se opta por medir el diámetro exterior con un reloj comparador Mitutoyo modelo ID-G112X, ajustando el cero del instrumento con un bloque patrón clase 0 (Mitutoyo) (Figuras 3 y 4) de dimensiones similares al diámetro estimado.

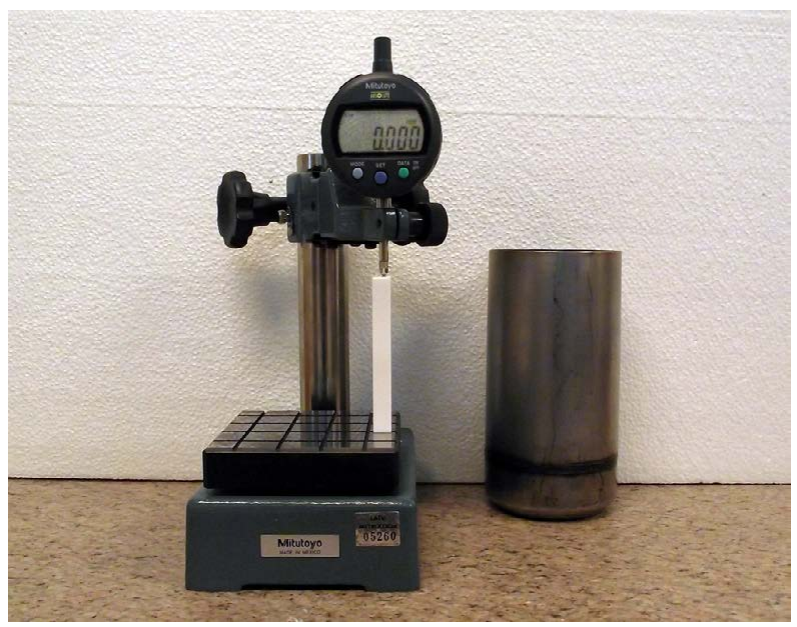

Figura 3. Ajuste de cero del comparador.

El espesor de chapa se mide con un micrómetro, intercalando en la cara interna del cilindro (campana) una esfera de acero de diámetro conocido (Figuras 5 y 6). 


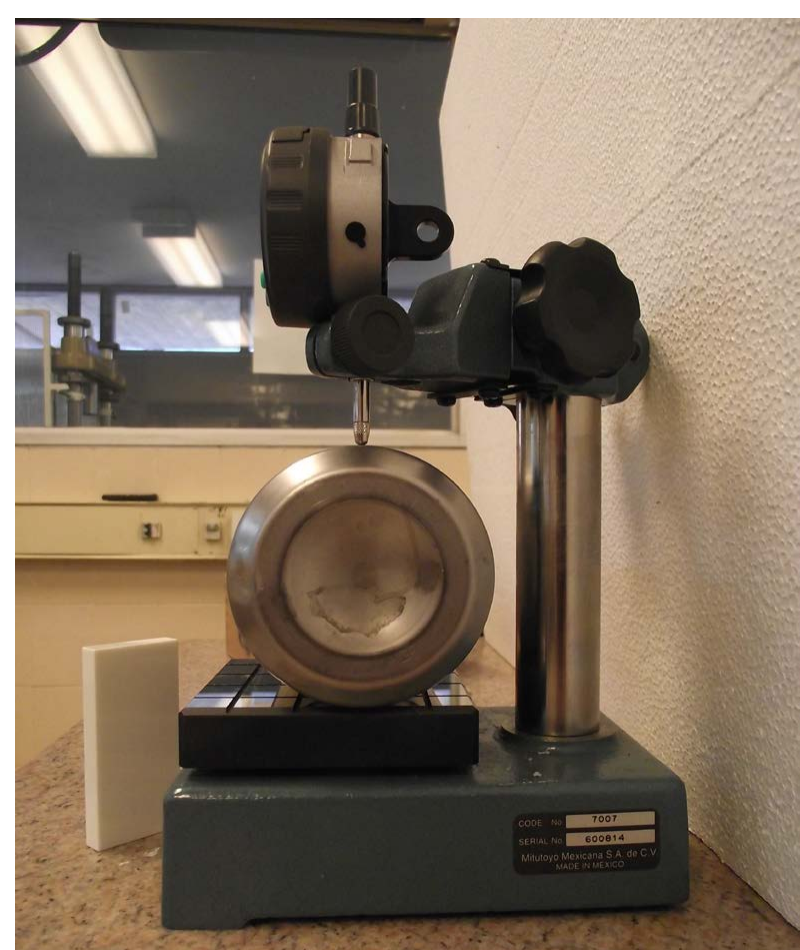

Figura 4. Medición del diámetro exterior de la campana.

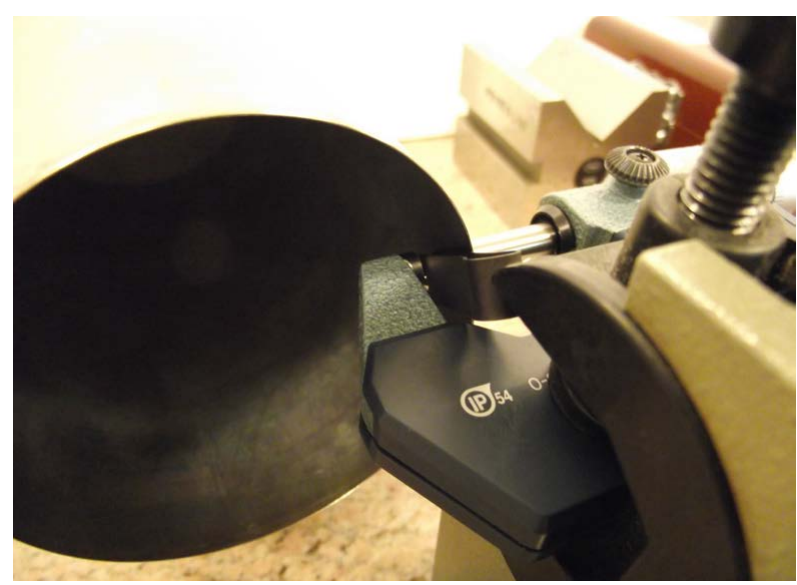

Figura 5. Medición del espesor de chapa.

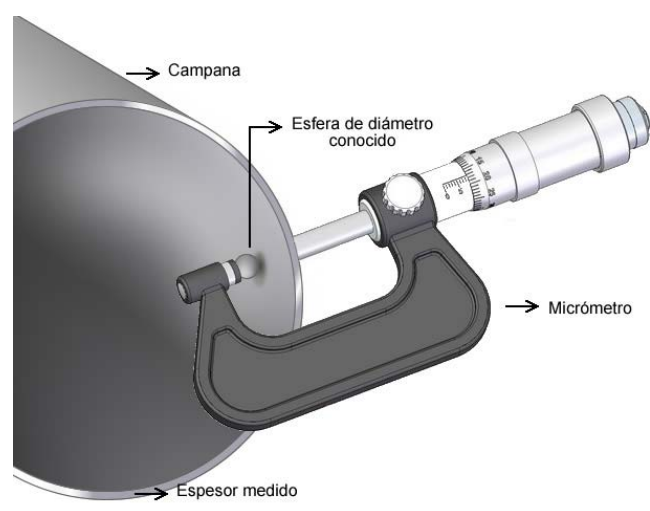

Figura 6. Diagrama de medición del espesor de chapa.

Para las medidas de masa se utilizará una balanza marca Mettler modelo PJ3000 con intervalo nominal $3000 \mathrm{~g}$ y divi- sión 0,02 g debido a su adecuado rango de medida, además de contar con dispositivo para pesar colgando del plato.

Validación de las mediciones. Para la prueba de funcionamiento y validación de resultados se calibra un módulo de presión diferencial FLUKE modelo 700P01 y se comparan los resultados con los obtenidos por otro NMI (Instituto Nacional de Metrología) con magnitudes declaradas en KCDB (Key Comparison Database) del BIPM (Oficina Internacional de Pesas y Medidas) para este intervalo.

Modelo matemático. La presión generada por un manómetro de campana invertida está dada por la siguiente ecuación (Zvizdic, et al., 2007):

$P=\frac{\left[\sum_{i}\left[m_{i .}\left(1-\frac{\rho_{a}}{\rho_{m i}}\right)\right]\right] \cdot g}{A_{0} \cdot[1+2 \cdot \alpha(t-20)]}$

Donde $A_{0}$ es el área efectiva a la temperatura de referencia, $g$ es la gravedad local, $\rho_{a}$ es la densidad de aire circundante y $\rho_{m i}$ la densidad de las masas en el punto $i$. $\alpha$ es el coeficiente de expansión térmica lineal de la balanza y el depósito, y finalmente $t$ es la temperatura de la balanza durante su uso.

La medida del área $\left(A_{0}\right)$, teniendo en cuenta el método de medida, se obtiene a partir del diámetro según:

$A_{o}=\pi\left(\frac{(D e-2 . E)}{2}\right)^{2}$

Donde $D_{e}$ es el diámetro exterior y $E$ el espesor de chapa. Para el cálculo de incertidumbre se tuvieron en cuenta las incertidumbres de calibración del micrómetro y comparador, repetibilidad, variación de temperatura, incertidumbre en la calibración de la esfera, resolución de los instrumentos y el componente de variabilidad debida al cambio en el eje del cilindro. Dado el pobre acabado del objeto utilizado como campana, la existencia de soldaduras y la falta de rigidez se obtuvo un valor de incertidumbre tipo A, más de $10^{3}$ veces mayor que el conjunto de componentes tipo B, por lo que en este prototipo estas últimas pueden considerarse despreciables.

\section{Resultados}

Se construyó el prototipo con las partes mencionadas. La Figura 7 muestra dos perspectivas del prototipo construido. En las fotos el instrumento está conectado al módulo de presión Fluke con el que se realiza la validación de las mediciones.
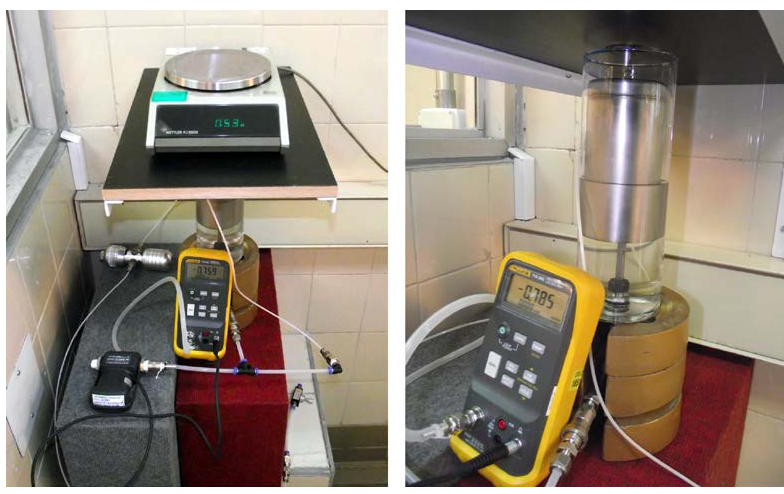

Figura 7. Balanza de campana conectada a un módulo de presión diferencial. 
Para las mediciones del diámetro de campana se realizaron 5 repeticiones de medidas en 3 diámetros girados $120^{\circ}$ entre sí a $1 \mathrm{~cm}$ aproximadamente del extremo cerrado de la campana. Las medidas a lo largo de la altura de la campana no se pudieron realizar; la fuerza de medida del reloj comparador deformaba significativamente la geometría del cilindro provocando errores importantes en el diámetro medido.

\section{Resultados de las mediciones}

Intervalo máximo de medición en la campana $=8 \mathrm{mbar}$. El poco peso de la campana y su distribución no permite el correcto funcionamiento más allá de este valor debido a que pierde verticalidad variando el área específica en un valor desconocido.

$\begin{aligned} & \text { Área específica } \\ & \text { de la campana }\end{aligned}=A_{0}=\left(4,3866 \times 10^{-3} \pm 3,9 \times 10^{-6}\right) \mathrm{m}^{2}$

Validación de mediciones. Se realiza la calibración de un sensor piezoresistivo marca FLUKE modelo 700P01 y se comparan estos resultados con los obtenidos por el CEM para el mismo instrumento.

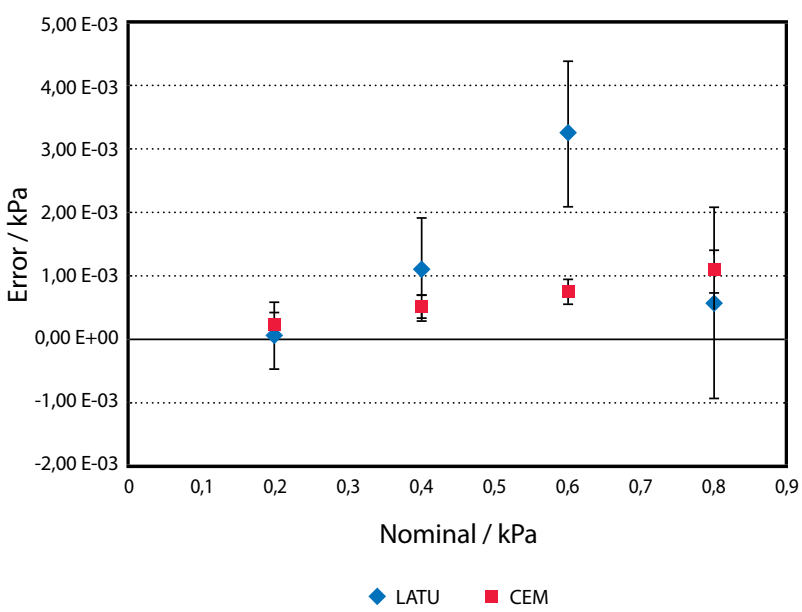

Gráfico 1. Resultados de calibración comparativos LATUCEM.

El análisis de errores normalizados arroja resultados concordantes en todos los puntos excepto el correspondiente a $0,6 \mathrm{kPa}$ que parece estar fuera de la tendencia. Una posible explicación de esta conducta irregular es que al utilizar cilindros de uso doméstico puede haberse dado un rozamiento entre la campana y el contenedor por defectos en la geometría de ambos.

\begin{tabular}{|c|c|}
\hline Nominal / $\mathbf{k P a}$ & Error normalizado \\
\hline 0,2 & 0,3 \\
0,4 & 0,7 \\
0,6 & 2,1 \\
0,8 & 0,4 \\
\hline
\end{tabular}

Tabla 2. Errores normalizados LATU-CEM.

Al aplicar una regresión lineal en ambas calibraciones puede verse que se obtienen resultados casi idénticos.

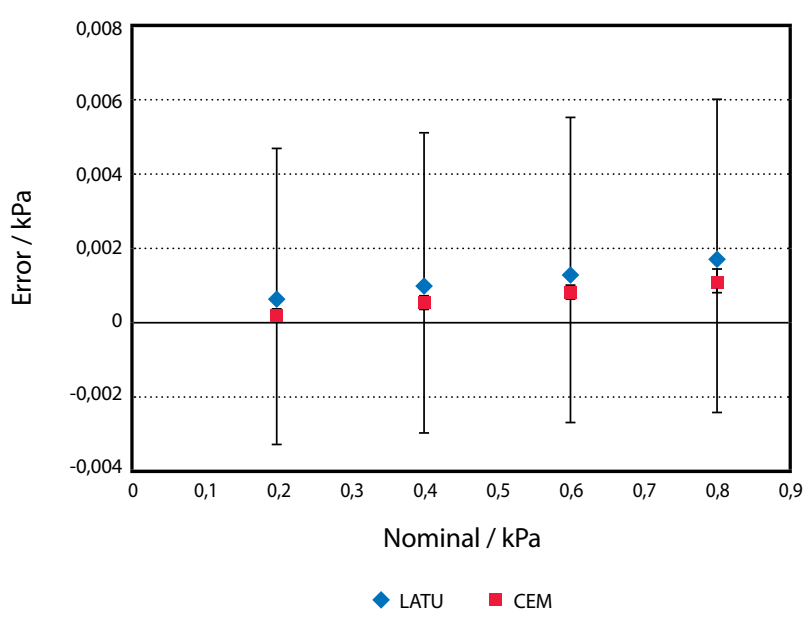

Gráfico 2. Resultados de regresión comparativos LATUCEM.

La contribución de incertidumbre aportada por la regresión en el caso de los resultados obtenidos con la campana invertida es significativa debido a la falta de linealidad de los puntos en la curva. Sin embargo, al amortiguar las diferencias con un ajuste lineal puede verse que la curva de calibración obtenida con el prototipo es casi idéntica a la obtenida por CEM.

\begin{tabular}{|c|c|c|c|c|}
\hline & \multicolumn{2}{|c|}{ Resultados LATU } & \multicolumn{2}{c|}{ Resultados CEM } \\
\hline Nominal / $\mathrm{kPa}$ & Error $/ \mathrm{kPa}$ & $\mathrm{U}_{\text {Error }} / \mathrm{kPa}$ & Error $/ \mathrm{kPa}$ & $\mathrm{U}_{\text {Error }} / \mathrm{kPa}$ \\
0,2 & $7 \times 10^{-05}$ & $5,3 \times 10^{-04}$ & $2,5 \times 10^{-04}$ & $1,2 \times 10^{-04}$ \\
0,4 & $1,09 \times 10^{-03}$ & $8,2 \times 10^{-04}$ & $5,0 \times 10^{-04}$ & $1,2 \times 10^{-04}$ \\
0,6 & $3,2 \times 10^{-03}$ & $1,1 \times 10^{-03}$ & $7,6 \times 10^{-04}$ & $1,2 \times 10^{-04}$ \\
0,8 & $6 \times 10^{-04}$ & $1,5 \times 10^{-03}$ & $1,11 \times 10^{-03}$ & $2,9 \times 10^{-04}$ \\
\hline
\end{tabular}

Tabla 1. Resultados de calibración comparativos LATU-CEM. 


\section{Conclusiones}

\section{Resultados}

Si bien el objetivo de este trabajo es solamente la construcción y prueba de un prototipo de bajo costo, los resultados obtenidos con el mismo son muy cercanos a los de una calibración anterior realizada por un NMI con capacidades reconocidas, por lo que el sistema diseñado se considera confiable.

\section{Características constructivas}

A continuación se enumeran algunas mejoras necesarias para el desarrollo de un equipo definitivo, de acuerdo a las observaciones realizadas durante el ejercicio con este prototipo.

- Masa de la campana. La masa de la campana elegida es insuficiente, lo que impidió utilizar el instrumento en todo el intervalo posible dado por la altura de la campana. Esto se debe a que cuando el empuje (ascendente) es cercano a la fuerza ejercida por la masa, el cilindro pierde estabilidad. Esto provoca la pérdida de la verticalidad afectando el valor de área específica expuesta. Es recomendable utilizar una masa al menos 5 veces mayor que la equivalente al empuje para el máximo intervalo posible.

- Diámetro. Las características dimensionales del elemento sensor son controlantes en la incertidumbre del instrumento, en particular el diámetro. Es deseable utilizar un cilindro con un acabado de precisión para minimizar el aporte de incertidumbre de esta magnitud. Por otra parte es conveniente utilizar un elemento sensor con mayor diámetro. Una mayor área específica permite una mayor precisión, menores incertidumbres relativas y no modifica el intervalo de lectura. Es recomendable un diámetro mayor a $15 \mathrm{~cm}$.

- Materiales. Si bien originalmente los elementos se diseñaban de acero inoxidable, estos no permiten ver el comportamiento del fluido dentro y fuera de la campana. Es conveniente, por tanto, evaluar la utilización de un material transparente. Esto permite además tener una referencia del desplazamiento del líquido, con lo que puede estimarse también la presión aplicada, aunque con menor precisión.

- Rigidez. El material de la campana debe ser suficientemente rígido, de modo que permita la calibración en diámetro a distintos ejes y alturas.
- Nivel. El nivel perfectamente vertical de la campana es crítico para la medida del área específica en servicio, por lo que se recomienda disponer de niveles y dispositivos de ajuste de nivel tanto en la campana como en el depósito para asegurar que trabajen alineados.

- Balanza. Las características metrológicas de la balanza utilizada se consideran adecuadas para este instrumento.

- Sistema general. Los resultados obtenidos demuestran que el diseño general propuesto para el prototipo es suficientemente preciso para realizar las mediciones propuestas, logrando el objetivo de diseñar un instrumento económico que no insume altos costos para mantener su trazabilidad y que puede ser calibrado en la institución.

\section{Continuación del trabajo}

Es recomendable la construcción de un instrumento final basado en el prototipo probado en este ejercicio que incluya las mejoras detalladas anteriormente.

\section{Reconocimientos}

Se agradece a todo el personal de Metrología Científica del LATU, quienes de una manera u otra tomaron parte en la discusión del modelo, selección de partes y su posterior construcción. También se extiende el reconocimiento al Sr. Ángel Flores Torres, colega del laboratorio nacional de presión de Chile (ENAER), quien nos proporcionó excelentes fotos y manuales de su balanza Junkalor de campana simple, lo que nos permitió ajustar mejor algunos aspectos de nuestro diseño.

\section{Referencias}

International Organization for Standardization, 2005. ISO/IEC 17025: General requirements for the competence of testing and calibration laboratories. Ginebra: ISO.

Rostron Hindley, H. A., 1947. Direct-reading differential micromanometer. En: Journal of Scientific Instruments, 24(11), pp.295-296.

Zvizdic, D., Grgec Bermanec, L., Schultz, A. y Veliki, T., 2007. Characterization of LPM diving bell manometer. En: IMEKO. IMEKO 20th TC3, 3rd TC16 and 1st TC22 International Conference Cultivating Metrological Knowledge. (Mérida 27 de noviembre a 1 de diciembre de 2007). Mérida: IMEKO. 